THÉMATA. Revista de Filosofía

$\mathrm{N}^{\circ} 50$, julio-diciembre (2014) pp.: 201-214

ISSN: 0212-8365 e-ISSN: 2253-900X

doi: 10.12795/themata.2014.i50.09

\title{
INTELECCIÓN Y CONCIENCIA EN HUSSERL
}

\author{
INTELLECT AND CONSCIOUSNESS IN HUSSERL
}

\author{
Urbano Ferrer ${ }^{1}$ \\ Universidad de Murcia (España)
}

Recibido: 29-04-2014

Aceptado: 27-06-2014

Resumen: Se delimitan cuidadosamente, siguiendo a Husserl, los distintos niveles de intelección, desde las unidades objetivas hasta los primeros principios que se hacen explícitos en los juicios. Pero un caso particular de intelección es la que se refiere a la conciencia propia, como unidad que persiste a través del flujo de las vivencias. Lo cual nos plantea el problema de la constitución de la temporalidad inmanente a la conciencia, así como la diferencia entre conciencia intencional y autoconciencia. Por último, se hace referencia a la distinción entre conciencia actual y habitual y el insuficiente tratamiento por Husserl de la segunda.

Palabras-clave: intencionalidad, objetividad, autoconciencia, pasividades, principios lógicos.

\begin{abstract}
Husserl carefully delimites the various levels of intellection from the objective unities to the first principles, made explicit in the judgements. A particular case of intellection is that which refers to the own consciousness as unity which accompanies the stream of experiences. That poses the problem of the constitution of the immanent temporality of the consciousness as well as the difference between intencional consciousness and selfconsciousness. Finally, we address the difference between actual and habitual conscioussness, concluding that the later is not sufficiently dealt with by Husserl.
\end{abstract}

Key-words: intentionality, selfconsciousness, passivities, logical principles.

[1] (ferrer@um.es ) Catedrático de Filosofía Moral en la Universidad de Murcia. Miembro fundador de la SEFE (Sociedad Española de Fenomenología) y de la AEP (Asociación Española de Personalismo). Profesor invitado en las Universidades de Dresde y Lublin. Condecorado por la Universidad de Murcia con la Medalla Alfonso X a la labor docente e investigadora. Entre sus libros relacionados con la Fenomenología se encuentran Qué significa ser persona (Palabra: Madrid) 2002; Desarrollos de Ética fenomenológica (Moralea: Albacete) 2003; Adolf Reinach: las Ontologías regionales (Cuadernos Anuario Filosófico: Pamplona), 2005; Welt und Praxis. Schritte zu einer phänomenologischen Handlungstheorie (Königshausen\&Neumann/Würzburg) 2006; La trayectoria fenomenológica de Husserl (Eunsa: Pamplona) 2008; en colaboración con S. Sánchez-Migallón, La ética de Edmundo Husserl (Thémata/Plaza y Valdés: Sevilla) 2011. Es editor de Para comprender a Edith Stein (Palabra: Madrid) 2008. Ha participado como ponente en Congresos celebrados en distintos países europeos e hispanoamericanos. Es autor de numerosos artículos en revistas especializadas de Filosofía. 


\section{Niveles en la intelección}

Ya desde la primera Investigación Lógica ocupa un lugar central en Husserl el acto de intelección, en su función de animar (beseelen) los signos expresivos, dirigiéndolos hacia el objeto aprehendido intelectivamente: fenomenológicamente es el acto de donación de sentido (Sinngebungsakt), esencialmente diferenciado de otros actos que lo pueden acompañar (la percepción, la intución imaginativa, los actos a los que incumbe notificar vivencias en el curso de la comunicación...), pero a los que no queda adscrito el significado por el que se reconocen aquellos signos. Análogamente, la percepción es un acto cuya dirección intencional está vuelta hacia un objeto presente él mismo, dado en persona. El hecho de que le correspondan unos datos sensoriales no exime de la necesidad de aprehender el objeto percibido con su configuración particular: así lo muestran en especial los casos de ambigüedad perceptiva, que solo se pueden resolver contando con la orientación por parte del acto aprehensor hacia uno u otro percepto. No basta, por tanto, con las leyes pasivas de la asociación (por contigüidad, semejanza, contraste...) de los datos sensoriales que sirven de apoyo al objeto intencional para la constitución del acto de percibir.

Pero es tras el aislamiento fenomenológico del yo en Ideen I cuando se destaca más patentemente esta función aprehensora del acto de percibir: "Percepción, en el sentido normal de la palabra, no quiere decir solo en general que alguna cosa aparece con personal presencia al yo, sino que el yo se percata de la cosa que aparezca, aprehendiéndola, poniéndola como realmente existente". ${ }^{2}$ Y paralelamente a la unidad del yo, que congrega sus distintos actos de esencia diversa, el objeto -o la configuración objetiva- aprehendido es también una unidad que rebasa los sentidos de aprehensión con los que lo mentamos intelectivamente, como a su vez los sentidos aprehensivos exceden también la base sensorial sobre la que se edifican. En efecto, el desnivel entre los datos sensoriales de partida y el sentido intelectivo de la aprehensión está marcado por el carácter universal del segundo, y por su parte el objeto trascendente a los sentidos de aprehensión -y que agrupa a estos-, sin ser él universal, viene mediado por la universalidad de las determinaciones que en condición de predicados adhieren a él.

Desde luego hay actos en los que la aprehensión del objeto se torna explícita a través de las determinaciones que le convienen: basta para ello con transformar los sentidos de aprehensión en predicados y el objeto que unifica los sentidos de aprehensión en sujeto lógico sobre el que recaen los predicados. De este modo, aparece como correlato de la aprehensión intelectiva la cópula "es", por deberse a ella la diferencia entre sujeto y predicados. Véase el correspondiente análisis fenomenológico de Husserl: "La palabra blanco mienta con

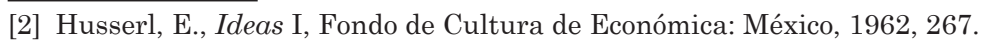

THÉMATA. Revista de Filosofía, Nº50 julio-diciembre (2014) pp.: 201-214 doi: 10.12795/themata.2014.i50.09 
seguridad algo en el papel blanco mismo y, por ende, coincide, en el estado del cumplimiento de este mentar, con la percepción parcial referente al momento blanco del objeto. Pero no basta advertir una mera coincidencia con esta percepción parcial... La intención de la palabra blanco sólo parcialmente coincide con el momento de color del objeto que aparece; queda en esto un resto de significación, una forma que no encuentra en el fenómeno mismo nada en que confirmarse. Papel blanco quiere decir papel que es blanco". ${ }^{3}$ Los actos expresamente intelectivos son, por consiguiente, actos fundados, tales que sobre el recubrimiento sensorial entre los datos hechos presentes -un papel presente a la conciencia y su color blanco- se monta un nuevo acto, que aprehende intelectivamente la unidad predicativa entre ser papel y ser blanco, unidad que es posible por la comunidad en el ser que les sirve de enlace.

Sin embargo, la unidad expresada con el verbo copulativo no se limita a las atribuciones simples, como la anterior, sino que abre un área en principio indefinida, ya que mediante el recurso a la nominalización una oración gramatical puede ser a su vez sujeto de nuevas determinaciones a un nivel metalingüístico. (1) "El papel es blanco es un enunciado afirmativo", (2) "Esto que has dicho (el enunciado 1) es verdadero", (3) "Las frases susodichas pertenecen a la lengua española"... Se produce con ello un crecimiento de la conciencia por sucesivos actos de intelección, bien que situados en el plano lógico-expresivo de las oraciones gramaticales. Es un crecimiento lógico por formalización, en vista de que los predicados pertenecen a un metalenguaje que formaliza el lenguaje directo o de primer grado en el que se encuentra su sujeto. La otra posibilidad de crecimiento lógico es por generalización, resultado de que el predicado ha de tener, según una ley lógica, mayor extensión que el sujeto. En este sentido, la generalización viene acompañada de la aserción de que "no todo b es a" cuando se dice "a es b". Tanto la formalización como la generalización son proseguibles in infinitum, ya que por parte de la mente nunca se alcanza el término mayor que el cual no cabe pensar otro. En cambio, si adoptamos como punto de partida regiones objetivas determinadas o unas traslaciones lingüísticas definidas, topamos con unos límites, en todo caso no debidos a la operación de entender.

Hemos encontrado, pues, un primer nivel de intelección de objetos en la significación y en la percepción y un segundo nivel de intelección formal, representado arquetípicamente por la cópula, pero que también cabe exponer mediante los otros enlaces lógicos, sean conjuntivo, disyuntivo, condicional..., combinables de diversos modos. Ahora bien, malinterpretaríamos esta distinción de niveles si los viéramos toto caelo separados, por cuanto no es posible una aprehensión de objeto si no es como sustantivo, como adjetivo..., por tanto revestido de un elemento informador; como tampoco cabe delimitar un abstracto si no es acompañándolo de algún elemento articulativo, tales como "un

[3] Husserl, E., Investigaciones Lógicas, Revista de Occidente: Madrid, 1976, 694-695.

THÉMATA. Revista de Filosofía, $\mathrm{N}^{\mathrm{o}} 50$ julio-diciembre (2014) pp.: 201-214 doi: 10.12795/themata.2014.i50.09 
pájaro" (en indeterminación debido a su extensión), "el pájaro" (determinado por su comprensión lógica) o "todo pájaro" (en la inmediatez de su aprehensión específica). En el sentido inverso, no son posibles los conectivos lógico-formales sin unos contenidos posibles y efectivos en los que se realicen; pues de lo contrario estaríamos ante unas reglas de juego estipuladas arbitrariamente, sin validez en sí mismas.

De aquí la aparición de dos conceptos-límite: las materias puras o nucleares, desnudas de todo revestimiento lógico-gramatical, y los elementos lógico-formales, que invisten a las materias, sin que las unas ni los otros se den aisladamente en función intelectiva (la intelección de las formas vacías por separado exigiría su nominalización y los predicados que se le adscribieran ya habrían de ser metalingüísticos). Entre ambos extremos se hallan las intelecciones, provistas de un contenido definido y acordes con unas leyes de derivación. "Las materias puras permiten en último término la referencia objetiva gracias a su conformación en distintos niveles; de suerte que cualquier formación de cualquier nivel presenta a su vez, en sus términos correspondientes, materias y formas relativas". ${ }^{4}$ Un ejemplo de materia pura o abstracta sería lo que tienen en común "semejanza" y "semejante" o "esperar" y "esperanza", algo que ni siquiera puede expresarse porque al expresarlo ya lo estaríamos encuadrando cuando menos en una forma gramatical.

Sin embargo, inteligir algo (noein) no es objetivarlo, aun cuando se incluyan en ello elementos formales. Más bien inteligir implica referir lo inteligido a principios, por lo que la intelección sólo se cumple a fin de cuentas en el juicio comparativo (tercer nivel de intelección) con lo que lo hace válido. Si la operación cognoscitiva más propia en Lógica es la inferencia, en Teoría del conocimiento, en cambio, ha de decirse que es en el juicio donde el conocer definitivo se alcanza y expresa. Así lo entiende Husserl en continuidad con el pensamiento clásico, y en este sentido tiene por insuficiente toda mecánica calculatoria que intente reemplazar el conocer por principios. En efecto, en sus orígenes griegos desde Platón el valor del conocimiento científico no se medía tanto por el éxito en las aplicaciones cuanto por el reconocimiento de unos principios de validez incondicionada en los enunciados de la ciencia. "Nada estaba más lejos de esa exigencia (de la ciencia) que poner la mira en una especie de operación meramente técnica, cuya ingenuidad contrasta al extremo con otra operación: la de la propia normación radical por principios... A las ciencias especiales independientes les falta comprender la unilateralidad fundamental propia de sus operaciones; les falta comprender que solo empezarán a captar teóricamente el pleno sentido ontológico de su respectiva esfera de objetos... cuando dirijan sus indagaciones hacia la universalidad del ser y su fundamental unidad". ${ }^{5}$

[4] Husserl, E., Lógica formal y trascendental, Anexo 1, FCE: México, 1962, 309.

[5] Husserl, E., o.c., 8.

THÉMATA. Revista de Filosofía, Nº50 julio-diciembre (2014) pp.: 201-214 doi: 10.12795/themata.2014.i50.09 
La unidad de la razón se acredita, pues, en unos principios unitarios inteligidos originariamente, que rigen en general para todos los campos particulares del saber humano. En este sentido se mueve la empresa husserliana de delimitar los distintos estratos de la Lógica formal (a saber, $1^{\circ}$, diversos principios lógicos enmarcados en una analítica de la inferencia; $2^{\circ}$, lógica de la distinción sintáctica; $3^{\circ}$, teoría de la multiplicidad combinatoria), para reconducirlos luego a los principios trascendentales antepredicativos, válidos para todo ente posible susceptible de ser juzgado. Con este giro subjetivo-normativo a partir de las valideces apofánticas se propone Husserl trasladar las derivaciones objetivas a las operaciones judicativas correspondientes, efectuables por cualquier sujeto posible. Así, en el caso del principio de contradicción su versión lógico-trascendental establece que "si un juicio puede adecuarse a lo dado, en el sentido de una evidencia de las cosas mismas, su contradictorio no solo está excluido a priori como juicio, sino que tampoco puede (para el sujeto que juzga) adecuarse de semejante manera, y viceversa" ${ }^{6}$ (con ello, como se advierte, transitamos del juicio lógico a la operación de adecuarse o juzgar). Y manteniéndonos en la capa analítica de la Lógica, pero tomada ahora en toda su generalidad, la flexión lógico-trascendental del principio de contradicción dirá: "la no contradicción en su sentido más amplio, que incluye cualquier consecuencia analítica, es una condición necesaria y suficiente para poder formular precisamente un juicio posible". ${ }^{7}$ Junto al principio lógico de contradicción Husserl hace mención del principio de tercio excluso, el modus ponendo-ponens o el tollendo-tollens...

Si de la Lógica pasamos a la Axiología y Práctica de la razón, la situación presenta semejanzas. Tampoco aquí cabe acertar a casu con el valor correctamente juzgado, o bien con el juicio práctico directivo de la acción. Pues lo específico del juicio práctico es la normatividad a priori, orientativa de la acción en un estadio previo a su realización, y no la acomodación a un estado de cosas ya dado, ni siquiera a un estado de cosas previsible en unas circunstancias dadas. "Las leyes éticas no son leyes de cosas para el actuar, en tal caso no cabría otro actuar que el que ellas exigen; rebasan más bien las leyes meramente empíricas o aprióricas para las conexiones posibles efectivas o ideales de la naturaleza, ya que no regulan el ser de las acciones como cosas, sino que entregan normas de un deber". ${ }^{8}$ En otros términos: la verdad de la acción debida no está decidida en sí al margen de su intelección, sino que incumbe a esta proponerla normativamente conforme a la fórmula del imperativo categórico husserliano: "actúa de acuerdo con la mejor ciencia y conciencia".

[6] o.c., 202. La aclaración entre paréntesis es mía.

[7] o.c., 224.

[8] Roth, A., Edmund Husserls ethische Untersuchungen, Martinus Nijhoff: La Haya, 1960, 34 (reinscripción de textos tomados de los Manuscritos).

THÉMATA. Revista de Filosofía, №50 julio-diciembre (2014) pp.: 201-214 doi: 10.12795/themata.2014.i50.09 
Pero la intelección también puede dirigirse a la propia conciencia, en los términos de un hacerse consciente de ella. ¿Cómo es esto posible, si la conciencia no es un objeto intencional, ni tampoco un acto particular, sino que más bien acompaña a todos los actos de conciencia? Por otro lado, la expresión "la propia conciencia" parece aludir a algo que no es la conciencia misma y que permite distinguirla de la ajena. Con ello enlazamos con el segundo apartado de esta contribución.

\section{2. ¿Intelección de la conciencia?}

Si la conciencia no está dada de modo objetivo ni tampoco designa un género de actos intencionales (ya que todos los actos intencionales son conscientes), el modo de llegar a destacarla fenomenológicamente Husserl sólo queda que sea en tanto que momento interno integrado en los actos conscientes. Es lo que designamos usualmente como momento del darse cuenta integrado en la conciencia intencional. "Todo acto es conciencia de algo, pero todo acto es también consciente. Toda vivencia es 'sentida', es 'percibida' inmanentemente (conciencia interna), aunque no sea, naturalmente, objeto de mención, objeto de posición, no sea mentada... Toda vivencia en sentido genuino es internamente percibida. Pero el percibir 'interno' no es una vivencia en el mismo sentido; no es percibido internamente de nuevo". ${ }^{9}$ Es claro que si la conciencia interna fuera una vivencia entre las otras, seríamos conscientes internamente de ella en una nueva vivencia, iniciando así un inviable regressus in infinitum; por ello, hace notar Husserl que se trata de un tener conciencia que a su vez no puede ser objeto intencional dado en otra vivencia, sino que forma parte de la conciencia en su estar vuelta intencionalmente o in directo a lo que no es ella. ${ }^{10}$

Avanzando un paso más, lo que se descubre en la conciencia interna es el único yo, que es idéntico pese a vivir en distintas vivencias. Hay efectivamente un paralelismo entre la conciencia que acompaña a todos sus actos y el yo que es advertido en todos ellos, y esto nos permite dar su plena concreción al momento consciente en los actos intencionales. Es el yo presente en acto en cada una de sus vivencias el que cobra en estas conciencia de sí mismo y las reconduce al ahora viviente en el que ellas se hacen conscientes. En la medida

[9] Husserl, E., Lecciones de Fenomenología de la conciencia interna del tiempo, Trotta: Madrid, 2002, 151-152.

[10] Parece verse en ello un intento de solución a la dificultad que le plantea la descripción temprana de la conciencia presente en la V Investigación Lógica, según la cual la conciencia interna se referiría a las vivencias actualmente presentes como sus objetos (segunda y fundamental acepción del término conciencia). "Recordemos el regreso infinito que se produce por la circunstancia de que la percepción interna es también ella misma una vivencia, o sea, necesita ella misma una nueva percepción a la cual es aplicable lo mismo, etc.; regreso que Brentano trató de evitar con la distinción entre dirección primaria y secundaria de la percepción” (Husserl, E., Investigaciones Lógicas, 482).

THÉMATA. Revista de Filosofía, ${ }^{\circ} 50$ julio-diciembre (2014) pp.: 201-214 doi: 10.12795/themata.2014.i50.09 
en que en Investigaciones Lógicas no se hacía explícito fenomenológicamente el yo, tampoco podía encontrar Husserl todavía los términos precisos con que describir la conciencia interna, incurriendo de modo prácticamente inevitable en la fórmula falaz de la conciencia objetivante. Pero no siendo el yo originariamente objeto para sí mismo, tampoco lo puede ser el ahora en el que se hacen conscientes sus vivencias en transcurso. La pretendida imagen objetiva del ahora como límite ideal no es sino una proyección espacializante que obstruye la mirada, al desviar la atención del ahora perteneciente al yo y que da vida a sus vivencias hacia lo que queda fuera. De un modo positivo lo describe Husserl: "Exactamente consideradas (las vivencias concretas), tienen en su concreción solo una fase absolutamente originaria, aunque siempre en flujo continuo, el momento del ahora vivo"11. Así se explica que no sea un ahora fijado en sus contenidos, pero ni siquiera en su duración como ahora, pues es una duración que viene determinada en sus límites en cada caso por el yo.

Si la conciencia es algo adjetivo en relación con las vivencias y sobre la base de ello en relación con el yo que vive en las mismas, induciría a confusión la sustantivación de la conciencia como sujeto. Quien está alegre no es la conciencia, sino aquel que se vive alegre conscientemente con mayor o menor intensidad, con tales o cuales variaciones en el curso de la alegría, con unos u otros motivos... La conciencia se limita a reflejar de modo inmanente lo que transcurre en el yo-sujeto. ${ }^{12}$ Sin embargo, cabe denominar también conciencia al flujo unitario de vivencias cuando es acotado en un intervalo determinado y diferenciado de otros intervalos propios o ajenos: "mi conciencia", "el rato que fui consciente"... Es cierto que ni aun en estos casos la conciencia es originariamente sujeto, porque lo es de alguien. Entre las acepciones del término conciencia que Husserl enumera en Investigaciones Lógicas menciona esta en primer lugar como corriente de vivencias del yo empírico, pero añadiendo que deriva del sentido segundo o intencional, el ya mencionado en la nota 10. Y posteriormente en las Lecciones de la Fenomenología de la conciencia interna del tiempo la retoma, pero ahora como constituyéndose a la vez que el flujo de vivencias. Ahora bien, si hay una derivación a partir de la conciencia interna, ¿cómo se opera esta ampliación de lo presente hasta cubrir una duración?; es más, ¿es propiamente una derivación la integración de la corriente temporal en la conciencia presente? Interrogantes semejantes valen para el flujo que se hace consciente en su incesante fluir, en tanto que

[11] Husserl, E., Ideas I, 178.

[12] Cf. lo que dice al respecto K. Wojtyla: "La consciencia no existe por sí misma como una especie de sujeto de los actos conscientes; no existe ni como un sustrato óntico que preexista de modo independiente ni como una facultad... De cuanto se ha dicho para caracterizar la consciencia resulta que toda ella se encierra en sus propios actos y en su especificidad consciente, con la que se une el carácter reflejo como algo distinto de la objetivación cognoscitiva" (K. Wojtyla, Persona y acción, Palabra: Madrid, 2011, 74).

THÉMATA. Revista de Filosofía, $\mathrm{N}^{\circ} 50$ julio-diciembre (2014) pp.: 201-214

doi: 10.12795/themata.2014.i50.09 
partimos de que la conciencia directa o primera es la dirigida transversalmente a los objetos intencionales ${ }^{13}$.

La dilatación del presente en orden a abarcar la alegría completa y sus declives, según el mismo ejemplo husserliano ${ }^{14}$, no podría tener lugar al modo bergsoniano, por engrosamiento de la bola de nieve, sino que es una remisión intencional del presente a lo que acaba de pasar o retención y otra prolongación intencional hacia lo que está por venir o protensión, hasta comprender el todo duradero hecho consciente (sea una alegría en curso, una melodía que se va oyendo o el sonido de la bocina del tren que se va perdiendo y a la vez renovando). Las intenciones del pasado y del futuro así entendidas no son tanto actos propios -como serían el recuerdo propiamente dicho y la anticipación de lo que no es en acto- cuanto éx-tasis acompañando al presente o momentos suyos constituyentes del pasado y el futuro respectivamente, a modo de vectores, haciendo posible la cola de cometa de las retenciones acumuladas y las expectativas todavía no despejadas o por cumplir. Otra precisión es que tampoco el presente vuelto extáticamente hacia el pasado y el futuro lo entiende Husserl como un acto expreso, sino que se trata del contenido de sensaciones inmanente a la conciencia en transcurso antes de ser animado intencionalmente por el acto (sea perceptivo o imaginativo); sin el presente dejaríamos de estar ante un contenido viviente para el yo consciente, que siempre es en presente ${ }^{15}$.

Retomemos la pregunta formulada más arriba, relativa al tránsito de la conciencia del presente vivo al flujo vivencial. ¿Cómo se efectúa? ¿Se trata de una derivación inmanente a la conciencia? La conciencia está polarizada en torno al yo y al objeto, a los que remiten respectivamente las noesis y los noemas, formando la correlación noesis-noema, en la que según Husserl está instalada la conciencia de modo continuo y renovado. ¿Acaso alguno de los dos polos posee connotación temporal? Si nos fijamos en el yo, su primera característica descriptiva es la permanencia inconmovible en presente a través de todas sus variaciones en las noesis o actos. "El yo parece estar ahí constantemente, incluso necesariamente, y esta constancia no es patentemente la de una vivencia estúpidamente obstinada, la de una idea fija". ${ }^{16} \mathrm{Ni}$ desaparece de sus

[13] "El flujo de la conciencia inmanente tempoconstituyente no solamente es, sino que se articula de una forma tan admirable, y no obstante tan comprensible, que en él necesariamente tiene que producirse un autoaparecer del flujo, y por ello el flujo mismo necesariamente ha de ser captable al fluir. El autoaparecer del flujo no requiere un segundo flujo, sino que como fenómeno se constituye en sí mismo" (Husserl, E., Lecciones de la Fenomenología de la conciencia interna del tiempo, 103). La derivación se advierte aquí en que en la autoaparición del flujo se está contando ya con la conciencia interna como autoaparición, extendiéndola al flujo.

[14] El mismo ejemplo es comentado con mayor amplitud por E. Stein, Ser finito y ser eterno, Obras III, Monte Carmelo: Burgos, 2007, Cap. III, 4, 682-685.

[15] El presente es lo que convierte en vivientes a los contenidos de conciencia, como bien refleja el título de la obra de Held, K., Lebendige Gegenwart, Martinus Nijhoff: La Haya, 1966.

[16] Husserl, E., Ideas, p. 132.

THÉMATA. Revista de Filosofía, Nº50 julio-diciembre (2014) pp.: 201-214

doi: 10.12795/themata.2014.i50.09 
actos ni se transforma en otro, sino que está presente para sí mismo sin que la temporalidad le afecte como foco constante de las vivencias. Mientras el tiempo queda en el orden del aparecer, el yo es, según la fórmula husserliana, una trascendencia en la inmanencia. Pero si atendemos al objeto, lo propio suyo es hacer converger los distintos noemas, que como escorzos perceptivos o bien como significados apuntan a él. Los contenidos de conciencia se van esbozando de modo inmanente, pero el objeto que los agrupa es el puro punto de unidad trascendente a los distintos apareceres. Por tanto, en ninguno de los dos polos encontramos la temporalidad del flujo de conciencia.

Queda por examinar el hacerse presente de los objetos en la conciencia. ¿Podría hallarse aquí la temporalidad? Comoquiera que la presencia a la conciencia es algo constante para todos sus objetos, no podría ser una presencia afectada de temporalidad. ${ }^{17}$ Según ello, tener conciencia objetiva del tiempo equivale a convertirlo en un abstracto (ya se trate de un día, cuatro horas, cinco minutos...), prescindiendo tanto de su fluencia como de la concreción temporal propia de una realidad determinada (según se dice en el Libro del Eclesiastés, el tiempo se ramifica en los distintos tiempos adecuados a cada actividad humana $\left.{ }^{18}\right)$. Por ello, la propuesta husserliana de incorporar a la conciencia un dinamismo de presentificación (Vergegenwärtigung) no se ve cómo pueda ser conjugada con la constancia y unicidad de la presencia mental.

A este respecto, pondría por mi parte las dos objeciones siguientes a Husserl: $1^{\circ}$, no es compatible el flujo incesante de la conciencia con el estar presentes a ella los objetos con constancia mental, ya que quedaría por acoplar el flujo a la conciencia que le es simultánea (Husserl califica esta duplicidad dentro del flujo, de la que no puede dar razón, como un hecho admirable, según se ha transcrito en la nota 13$) ; 2^{\circ}$, el flujo habría de precederse a sí mismo para que la conciencia de objeto se insertara en él, en tanto que la conciencia objetiva no es una fase más en el monótono fluir, sino que lo es del mismo fluir en su conjunto; en otros términos: no solo hay sucesión de fases, sino identificación previa del objeto (trátese de una melodía, la bocina del tren o la aparición visual de una llanura), para que este pueda aunar los distintos momentos pertenecientes a él. Pero si en vista de estas dificultades referimos el tiempo inmanente solo a los contenidos hyléticos de conciencia, como admite Husserl, entonces disociamos estos contenidos del objeto, dejándolos faltos de su eje sustentador.

[17] Es un aspecto destacado insistentemente por L. Polo en los 4 volúmenes de su Teoría del conocimiento. Sobre el modo de hacer compatibles la presencia mental y la temporalidad a través de los abstractos como articulaciones presenciales del tiempo, me he ocupado en Ferrer, U., "Ampliaciones de la noción fenomenológica de mundo en L. Polo", Studia Poliana, 5 (2003), 263-280.

[18] Eclesiastés, 3.1.

THÉMATA. Revista de Filosofía, $\mathrm{N}^{\circ} 50$ julio-diciembre (2014) pp.: 201-214

doi: 10.12795/themata.2014.i50.09 


\section{Modalidades de autoconciencia}

La conciencia se nos ha presentado como actividad de un sujeto o yo consciente, que versa sobre un término distinto de ella misma. Tal esquema no es aplicable sin más a la autoconciencia, a no ser que le adjuntemos algún elemento pasivo en la vivencia de sí mismo. Tales son las modalidades de reflexividad originaria con terminología de A. Millán-Puelles, en las que la subjetividad es vivenciada por ella misma, ya sea en la opresión por el dolor, en el ser reclamada por alguna necesidad mediada por el cuerpo, en el requerimiento que le hace el deber moral o en la vivencia de sí misma por el alter ego. ${ }^{19}$ En todos estos casos no se trata de un desdoblamiento interno a la conciencia, sino más bien de un adquirir conciencia de sí estando motivada por lo que no ha sido constituido por ella como objeto. Husserl toma en cuenta ciertamente las pasividades originales en el yo consciente. Pero, ¿se entienden desde ellas las correspondientes formas de autoconciencia?

Un primer nivel de pasividades son las que proceden del cuerpo y desde él son incorporadas a la subjetividad, la cual se vive a sí misma en ellas. Así, el hambre, la fatiga o el sueño no conciernen meramente a mi cuerpo, sino que me acucian como ser corporal, haciéndolas propias y expresándolas en términos de querer motivado. Son necesidades que se presentan a la vez como carencias y como exigencias ${ }^{20}$ que el agente ha de encauzar. Reúnen, pues, las dos condiciones susodichas para dar lugar a la autoconciencia: la pasividad anterior a la conciencia y la integración activa y consciente en el sujeto. En un sentido más amplio, cada vez que la situación social e histórica me motiva en uno u otro sentido se conjugan la pasividad de lo que me precede y la respuesta consciente del yo, que son de rigor para poder hablar de autoconciencia. P. Ricoeur ve en ello la superación definitiva por Husserl del naturalismo e historicismo de la conciencia y, a la inversa, la incorporación del cuerpo y de lo pasivo en general a la subjetividad consciente. "Este reconocimiento de una subjetividad que no es acto, espontaneidad, que se constituye como pasividad, tendencia afectiva o hábito es del más alto interés; marca el primer esfuerzo de Husserl por instituir una experiencia total de la subjetividad en la que lo involuntario y el cuerpo propio no son abandonados a una explicación naturalista, sino recuperados a su subjetividad vivida". ${ }^{21}$

[19] Millán-Puelles, A, La estructura de la subjetividad, Rialp, Madrid, 1967, 346-363.

[20] En castellano el término "necesidad" no tiene la connotación subjetiva que posee en otros idiomas (al diferenciar entre nécessité y besoin en francés, o entre Notwendigkeit y Bedürfnis en alemán, o entre necessity y need e inglés), por lo que no resulta tan fácil percatarse del carácter psíquico-motor de la necesidad, si lo contraponemos al sentido constringente de la necesidad lógica o de la necesidad moral.

[21] Ricoeur, P., "La motivation comme loi fondamentale du monde de l'esprit", À l'école de la phénoménologie, Vrin: Paris, 2004, 145.

THÉMATA. Revista de Filosofía, N50 julio-diciembre (2014) pp.: 201-214

doi: 10.12795/themata.2014.i50.09 
Pero se da también un segundo estrato de pasividades reveladoras de la autoconciencia, a las que Husserl identifica como habitualidades del agente: sobrevienen cada vez que tomo una decisión, cada vez que me pronuncio en un sentido determinado..., ya que de ahora en adelante soy para mí el que se ha decidido de tal modo o quien ha adoptado el correspondiente pronunciamiento. La autoconciencia es aquí posible como conciencia de alguien que está ya definido por unos actos que ha realizado y que han quedado en él.

Análogamente, en un contexto ético puedo unificar "mi vida" al aprobar o desaprobar un fragmento suyo como "lo que ha transcurrido hasta tal momento" y que ahora -al objetivarlo- asumo como mío conscientemente, imprimiéndole un nuevo rumbo. Lo que objetivo es un trayecto de vida consciente, transformándolo mediante la nominalización en sujeto de nuevos predicados éticos, como vía para el crecimiento moral de la conciencia. Hay textos de la serie Kaizo ("Renovación") especialmente significativos: "Decisiones volitivas que ya había tomado, puede el hombre reconocerlas en afirmaciones volitivas o rechazarlas en negaciones volitivas; y lo mismo a propósito de acciones ya realizadas por él. La secuencia de hechos que han traído algo a la realidad no puede, claro está, desmontarse retrospectivamente. Pero el yo sí puede someter a crítica volitiva posterior la voluntad que ha actuado y que en la vida ulterior del yo prolongaba su vigencia de manera natural; puede, pues, confirmar tal vigencia o puede con un 'no volitivo' denegar está validez práctica". ${ }^{22}$

Pero, ¿de dónde esta necesidad de recapitulación y elevación sobre lo antes vivido? Desde el ángulo gnoseológico que nos guía, es justo la pérdida de algunos de los caracteres que dan plenitud al noema actualmente vivido lo que hace preciso sobreponerse a ella con el juicio de conjunto, que desde la nueva perspectiva asuma los noemas modificados y les dote del índice de la certeza de la creencia que con la modificación habían perdido, al menos en parte. Así pues, el contrapunto al crecimiento en los actos de conciencia está en el decaimiento de la propia conciencia mediante lo que Husserl llama intencionalidades noemáticas, como inflexiones de la certeza plenaria de la conciencia perceptiva originaria.

Sucede, así, que los noemas o correlatos objetivos declinan de su plenitud desprendiéndose de alguno de los componentes con que se integraban en la percepción, como son el índice de creencia que posee lo percibido (ausente en la imaginación), el carácter originario de la percepción ahora desactivado (cuando se trata de un recuerdo o rememoración) o el correlato colmado de la percepción, al que se vacía de todos sus aspectos intuitivos, mencionándolo sin más (tal es lo que ocurre en la intención significativa). Son expresiones de pasividad, por cuanto decaen de una actividad intencional primera más

[22] Husserl, E., Renovación del hombre y la cultura, Anthropos: Universidad Autónoma Metropolitana de México, 2002, 25. 
lograda. Husserl las compara con un cierto espejamiento de las formas activas de intencionalidad, según círculos que van estando cada vez más alejados del centro. Pero la objetivación de la conciencia que de este modo resulta de las anteriores modificaciones en los actos de conciencia, es inseparable del telos al que apuntan en su haber sido rebajadas de él: tal telos reside en el límite ideal de la percepción presente, la cual, sin embargo, en su forma adecuada o perfecta es inviable intencionalmente en lo que se refiere a la conciencia de sí o autopercepción.

\section{Conciencia habitual de sí mismo}

En su obra tardía Husserl repara en que la conciencia que el yo adquiere de sí no es la del yo vacío, como mero punto de convergencia de los actos cumplidos, sino la de un yo definido, que tiene un estilo destacado de comportarse, forjado por él y por el que se le reconoce. Nos preguntamos, sin embargo, si concede a los hábitos innatos y adquiridos todo el peso que les corresponde en el conocimiento. Es una tesis de Tomás de Aquino la de que cada alma se conoce de modo habitual por sus actos. ${ }^{23}$ ¿En qué términos está planteado por Husserl este conocimiento directo del propio yo?

Los hábitos en el sentido de habitualidades son determinaciones acumuladas por cada yo, una vez que ha realizado los actos y tomas de posición correspondientes. Tienen un carácter derivado e integran el conjunto de predicados que convienen al yo como sujeto lógico. Por tanto, lo que parece excluido de este planteamiento husserliano de los hábitos es un sí mismo previo a los actos y que se conociera habitualmente en sus actos; derivadamente, tampoco cabría que este sí mismo fuera materia para la configuración del yo mediante sus actos libres, a la manera como Edith Stein lo tiene en cuenta. ${ }^{24}$ También Ingarden ${ }^{25}$ ha advertido -desde la consideración del fenómeno de "tener responsabilidad"- lo que considera una limitación en Husserl en el modo de abordar el yo y su autoconocimiento.

[23] "Quantum ad actualem cognitionem, qua aliquis considerat se in actu animam habere, sic dico quod anima cognoscitur per actus suos... Ad cognitionem habitualem, sic dico quod anima per essentiam suam se videt id est, ex hoc ipso quod essentia sua est sibi praesens, est potens exire in actum cognitionis sui ipsius" (Tomás de Aquino, De Veritate, 10, 8 c)).

[24] "El ser anímico no se agota en la actualidad de su yo... El hombre es determinado en su integridad por los actos puntuales de su yo, es 'materia' para la formalización efectuada por la actividad del yo. Aquí nos encontramos con el sí mismo que puede y debe ser formalizado por el yo" (Stein, E., La estructura de la persona humana, BAC: Madrid, 1998, 149).

[25] "He de subrayar que tanto en la consideración de tener la responsabilidad cuanto en la del asumirla, no puede uno limitarse al yo puro y a las vivencias puras... Un puro vivir la acción que no se ejecutara en verdad realmente no bastaría en manera alguna para tener responsabilidad. La responsabilidad del agente brota tan sólo de la realidad del acto. Y un yo puro sin propiedades como el que Husserl propuso en un principio no podría realizar el acto ni tener responsabilidad por él, de modo que surgiera del carácter de la persona y fuera motivado y determinado por él" (Ingarden, R., Sobre la responsabilidad, Caparrós: Madrid, 2002, 60-61).

THÉMATA. Revista de Filosofía, №50 julio-diciembre (2014) pp.: 201-214 doi: 10.12795/themata.2014.i50.09 
Por otro lado, también se encuentra en Husserl el hábito activo, como unificador de distintas voliciones actuales y posibles. Es el sentido de hábito finalista, que integra, junto con los fines propuestos en acto, otros fines más indeterminados, pero sin los que los primeros no serían operativos. En este caso se trata de hábitos no como sedimentaciones de actos previos, sino tales que nos llevan a reconocer una teleología ya dada, reveladora en alguna medida de lo que cada hombre natural y personalmente es. "La serie indefinida de objetivos, fines y tareas debe estar conexa, pues si no el yo no sería un yo, no motivaría la consecución, el cumplimiento de una primera tarea, una nueva y así sucesivamente". ${ }^{26}$ Aquí se trata de una dirección finalista no puesta por el yo, sino su-puesta en los actos de querer y de la que solo cabría tener un conocimiento innato habitual, sin que Husserl lo afirme en términos suficientemente definidos.

Así parece sobreentenderlo en textos como el siguiente: "Al comienzo de la experiencia no hay todavía ningún sí mismo constituido que esté predado, disponible como objeto. Está enteramente oculto para él mismo y para los otros, al menos en la intuición". ${ }^{27} \mathrm{Al}$ decir que el conocimiento de sí mismo no está dado para sí mismo al menos en la intuición, deja abierto otro modo de conocimiento -el habitual- para el propio yo que preceda a la realización de sus actos y tomas de posición. Este conocimiento habría de tener estructura finalista para que se pudieran integrar en él las conexiones motivacionales y de medios a fines aludidas en otros textos, como el citado más arriba. He aquí algún texto más explícito sobre el hábito innato correspondiente al yo: "El yo como persona lleva en sí (como desprendible cognoscitivamente de él, aunque no sepa nada de ello) la idea de su ser persona como persona verdadera (la idea de su existencia auténtica, de su auténtico existir)". ${ }^{28}$

En conclusión: Husserl pone el énfasis en mayor medida en los hábitos adquiridos, definidores de una personalidad y originados a partir de los actos propios, que en el hábito impreso desde el inicio, relativo a la individualidad que cada cual es, por más que haya algunas alusiones a él en función de la teleología no puesta, que se revela en los propios actos y en el curso de la existencia conjunta, y ello en coherencia con la individualidad irreductible de toda existencia auténtica. Este segundo motivo pondría en la vía de una Antropo-

[26] "Die unendliche Kette von Zielen, Zwecken, Aufgaben kann aber nicht zusammenhanglos sein, sonst wäre das Ich nicht ein Ich, sonst motivierte nicht eine Erzielung, die Erfüllung einer ersten Augabe, eine neue und so fort" (Husserl, E., "Fichtes Menscheitsideal", AV (1911-21), Husserliana XXV, 1987, 1987).

[27] "Im Anfang der Erfahrung ist noch kein konstitutiertes 'Selbst' als Gegenstand vorgegeben, vorhanden. Es ist völlig verborgen für sich und für Andere, wenigstens in der Anschauung" (Husserl, E., Ideen II, Husserliana IV, 1952, 253).

[28] "Das Ich als Person trägt in sich (als aus ihm herauserkennbar, auch wenn es darum nicht weiss) Die Idee seines Seins als wahrer Person (die Idee seiner echten Existenz, seines echten Daseins" (Husserl, E., Zur Phänomenologie der Intersubjektivität II, Husserliana XIV, 1973, 297).

THÉMATA. Revista de Filosofía, №50 julio-diciembre (2014) pp.: 201-214 doi: 10.12795/themata.2014.i50.09 
logía teológica, por cuanto es Dios quien llama al hombre a su existencia y al final de los tiempos, según el Apocalipsis ${ }^{29}$, le confirma quién es. Terminaremos con otro texto sobre la individualidad irrenunciable de cada persona: "Lo que en sentido propio denominamos yo es una individualidad personal... El polo del yo no tiene solo sus cambiantes sedimentaciones, sino a través de los cambios en ellas una unidad constituida con estilo propio. El yo tiene su individualidad, su carácter individual de conjunto, que atraviesa de modo idéntico todas sus decisiones y su correspondiente estar decidido; como carácter individual tiene sus particularidades, sus propiedades singulares, a las que llamamos propiedades de carácter".$^{30}$ En esta individualidad y en el modo de llegar a ella no está diferenciada la que es debida a los hábitos adquiridos de la que previamente singulariza de modo innato a cada persona, confirmándose la segunda en la que es forjada o adquirida con los actos propios definidos.

[29] "Al vencedor daré el maná escondido y le daré una piedrecita blanca, y en la piedrecita escrito un nombre nuevo, que nadie conoce sino el que lo recibe (Apoc., 2, 17).

[30] "Vielmehr, was wir im eigentlichen Sinn Ich nennen meint eine personale Individualität... Der Ichpol hat nicht nur seine wechselnden Niederschläge, sondern durch ihren Wechsel hindurch eine in diesem Stil konstituierte Einheit. Das Ich hat Seine Individualität, seinen individuellen Gesamtcharakter, der durch alle Entscheidungen und Entscheidenheiten identisch hindurchgeht; als Individualcharakter hat er Besonderheiten, Sondereigenschaften, die Charaktereigenschaften heißen" (Husserl, E., Phenomenologische Psychologie, Husserliana IX, 1968, 215). 